\title{
Narrativa
}

\section{Gli scrittori, la critica e l'ordine spontaneo dell'economia moderna. Una conversazione con Federico Morganti}

\section{Stefano Adamo}

\section{CpenEdition}

\section{Journals}

Edizione digitale

URL: https://journals.openedition.org/narrativa/295

DOI: 10.4000/narrativa.295

ISSN: 2804-1224

\section{Editore}

Presses universitaires de Paris Nanterre

\section{Edizione cartacea}

Data di pubblicazione: 1 décembre 2020

Paginazione: 179-191

ISBN: 978-2-84016-381-7

ISSN: $1166-3243$

Notizia bibliografica digitale

Stefano Adamo, «Gli scrittori, la critica e l'ordine spontaneo dell'economia moderna. Una

conversazione con Federico Morganti», Narrativa [Online], 42 | 2020, online dal 01 décembre 2021

consultato il 08 décembre 2021. URL: http://journals.openedition.org/narrativa/295 ; DOI: https:// doi.org/10.4000/narrativa.295

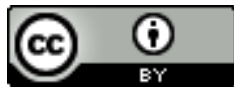

Narrativa est mise à disposition selon les termes de la Licence Creative Commons Attribution 4.0 International. 


\section{Gli scrittori, la critica e l'ordine spontaneo dell'economia moderna. Una conversazione con Federico Morganti}

$\mathrm{F}$ ederico Morganti è dottore di ricerca in filosofia, ha all'attivo diversi saggi, traduzioni e curatele scientifiche, scrive regolarmente sulla pagina culturale del quotidiano Il Foglio e collabora con pubblicazioni online. Dopo un periodo trascorso in Inghilterra come visiting scholar presso l'Università di Cambridge, è tornato a vivere a Roma dove lavora nel settore della comunicazione strategica. La sua storia di intellettuale trentenne che oltre a dedicarsi all'attività pubblicistica si specializza in una professione del terziario avanzato è simile a quella di molti uomini e donne della sua generazione che dopo un percorso formativo finalizzato all'insegnamento o alla ricerca hanno imboccato una strada professionale diversa da scuola e università. Secondo la teoria esposta in un recente ma già celebre saggio di Raffaele Alberto Ventura, egli sarebbe un esponente della "classe disagiata", una categoria in cui rientrerebbero quei nati negli anni Settanta-Ottanta il cui percorso professionale si discosta, magari solo apparentemente, da quello formativo e in cui è possibile riconoscere i personaggi di molta narrativa contemporanea sul lavoro.

Nel 2018, ha tradotto La guerra degli intellettuali al capitalismo, di Alan S. Kahan, uscito all'inizio dell'anno successivo per IBL libri. Il volume ripercorre la storia del secolare antagonismo fra attività intellettuale e attività mercantili o, per dirla col titolo originale, Mind vs. Money: un conflitto che fra mutue incomprensioni e atteggiamenti censori attraversa l'intera storia del pensiero occidentale, dalla condanna aristotelica della crematistica alle diverse declinazioni della critical theory contemporanea. Unica eccezione l'età dei lumi, quando intellettuali fra i più influenti, soprattutto in Gran Bretagna e negli Stati Uniti, abbracciarono i principi della società commerciale individuando in essi un sostegno materiale al nascente, e dunque ancora fragile, ideale di stato di diritto e un baluardo ideologico contro l'assolutismo politico. 
Nel primo saggio di questo volume, William Jackson ha raccontato la storia del progressivo allontanamento delle scienze economiche dai metodi e dalle modalità espressive del sapere umanistico, fenomeno che si è affermato soprattutto negli ultimi due secoli. In questa conversazione con Federico Morganti, proviamo a ragionare sul tema del difficile rapporto fra economia e discipline umanistiche soffermandoci sulle seconde. Chi si occupa di letteratura del lavoro o letteratura industriale, sia in senso creativo che in senso critico, tende spesso a individuare nella natura stessa della società commerciale l'origine dei problemi che affliggono i personaggi dei romanzi come anche degli aspetti più sgradevoli degli ambienti che vi sono evocati. Peraltro, laddove la letteratura, nella sua propensione a catturare il reale nella sua complessità, non risulti sufficientemente esplicita, è spesso la critica a indicare nel capitalismo, variamente declinato e con diversi prefissi, la diagnosi del malessere che nei testi si rappresenta ${ }^{1}$.

Federico Morganti interviene frequentemente nel dibattito pubblico in difesa dei principi alla base dell'economia di mercato. Pensando al volume che ha tradotto, chiedo a Morganti se l'ostilità che si riscontra nei confronti della società commerciale fra molti intellettuali italiani sia da ascrivere a una posizione prettamente filosofica, o non piuttosto a un giudizio maturato alla luce della storia specifica del capitalismo italiano.

\section{FEDERICO Morganti}

Kahan non offre approfondimenti specifici sul nostro paese, se non per quanto riguarda la fase fascista. Tuttavia, ritengo che, se seguissimo lui, dovremmo rispondere nel primo modo. Il testo di Kahan fa risalire l'ostilità degli intellettuali nei confronti del mercato addirittura alla filosofia greca. Già in Platone e Aristotele si trovano motivi di ostilità nei confronti del commercio e del denaro. Per Aristotele, ad esempio, il commercio va bene solo finché l'accumulazione di denaro non diventa un fine in sé (qualsiasi cosa ciò voglia dire, e non è per nulla scontato). Allo stesso tempo ci sono delle peculiarità tutte italiane. Chi condivide un orizzonte di pensiero liberale fa molta fatica a trasmettere l'idea che ciò che chiamiamo "società commerciale", il mercato, interagisce con una serie di fatti storici contingenti, perlopiù politici. E quindi da un lato, sì, l'Italia è certamente

1. Chi legge non avrà difficolta a riconoscere dietro queste parole numerose espressioni presenti anche in questo volume, come "orizzonte di edonistico sfruttamento," "epoca della precarietà", "sete infinita di profitto del capitale", "àncora di salvezza per la società capitalista", "valori trasformati in merce", e molte altre. Espressioni usate il più delle volte con la disinvoltura di chi presume di evocare nozioni di senso comune. 
un paese capitalista dove la libertà di commerciare e fare impresa è garantita, ma questa libertà incontra forti ostacoli nella regolamentazione (tassazione, limiti all'accesso alle professioni, vincoli di vario genere all'attività imprenditoriale), cioè nelle decisioni del legislatore. $\mathrm{E}$ in effetti li incontra già nella nostra Costituzione. Se non siamo sempre in grado di apprezzare i benefici della società commerciale, non è detto dipenda da difetti intrinseci di quest'ultima. Purtroppo, i liberali non sempre sono riusciti a trasmettere questo concetto.

Un libro che prova esattamente a far questo è La verità, vi prego, sul neoliberismo ${ }^{2}$ di Alberto Mingardi. Mingardi si rivolge proprio a quel tipo di pubblico che non vede di buon occhio il neoliberismo, e lo fa con grande pazienza e anche con grande bravura. Spesso c'è invece la tendenza contraria. Si tende a parlare agli anticapitalisti con il seguente tono: "Non avete capito niente, per tutto questo tempo vi siete sbagliati, il neoliberismo è un sistema perfetto e se a voi così non risulta è perché non capite niente di economia, o di come va il mondo". Bisognerebbe provare a mettersi nei panni di chi ascolta. Dietro all'anticapitalismo ci sono idee discutibili ma esigenze pienamente comprensibili, se non condivisibili (uguaglianza, tutela dell'ambiente, inclusione sociale). Occorre mostrare che queste battaglie possano essere rilette con gli strumenti del pensiero liberale e della società commerciale.

Per ritornare alla seconda parte della sua domanda, sulla specificità della storia del nostro paese, sappiamo che c'è stata una prima fase di boom economico post-bellico, seguita da una fase di centralizzazione della politica segnata da iniziative welfariste, di spesa pubblica (in particolare pensionistica) che si è accumulata a dismisura finendo per gravare sulle nuove generazioni. Tenderei a escludere si tratti di una vicenda soltanto italiana, perché le sue fasi sono abbastanza prevedibili. Dopo la guerra c'è un'economia che deve ripartire, qualcuno arriva e propone una serie di riforme per rilanciare un paese allo stremo. Si crea una fase di benessere, il cosiddetto "boom". E questo, paradossalmente, diventa il terreno fertile per la proliferazione delle dottrine anticapitaliste. Quando un paese è ridotto in macerie le persone hanno altro a cui pensare: c'è un'economia da far ripartire, imprese da far funzionare, ci si rimboccano le maniche. La generazione successiva, invece, può godere dei frutti del benessere creato dalla precedente, dedicare tempo allo studio e questo significa una nuova classe di intellettuali che ha la libertà di riflettere criticamente sul presente. Così è andata anche negli Stati Uniti negli anni '60 e '70, con la controcultura, dove un'intera generazione si è ribellata nei confronti dei genitori, cioè di coloro che avevano creato quel

\section{Mingardi Alberto, La verità, vi prego, sul neoliberismo, Venezia, Marsilio, 2019.}


benessere di cui ora i giovani potevano godere. E probabilmente lo stesso è accaduto anche in altri paesi europei che avevano sofferto la guerra e hanno conosciuto in seguito la controcultura (Francia, Germania). È un fenomeno che si vede molto bene nella musica di quegli anni, ma forse sto divagando.

\section{STEFANO ADAmo}

Facciamo pure una digressione sulla musica degli anni Sessanta e Settanta: perché la musica di quegli anni racconterebbe una svolta generazionale?

F.M.

La musica rock nasce alla metà degli anni ' 50 come grido di una generazione che rifiuta lo stile di vita dei genitori. All'inizio fu qualcosa di simile al capriccio di uno studente di scuola superiore, non esattamente un fenomeno "intellettuale". Il fenomeno si sarebbe intellettualizzato negli anni successivi, con una riflessione (forse non sempre matura) sulla civiltà occidentale, sui valori, sulla guerra e naturalmente sul capitalismo. La critica al capitale non sempre è esplicita; si tratta più che altro di un generale rifiuto dell'establishment (politici, industriali, le cosiddette "élite"). Il paradosso, in tutto questo, è che la generazione di musicisti e intellettuali della controcultura ha potuto dedicarsi alla musica e alla riflessione sui valori grazie al benessere che i genitori avevano messo loro a disposizione.

\section{S.A.}

Come sottolinea Hobsbawm nel Secolo breve, quella è stata infatti la prima generazione di giovani in Occidente ad aver potuto posporre l'ingresso nel mondo del lavoro senza rinunciare a una capacità di spesa che fino ad allora era stata una prerogativa di chi era economicamente autosufficiente.

F.M.

Esatto. Questo significa avere tempo da investire nello studio, anche musicale, fosse pure da autodidatti. Potevano ingenuamente immaginare una sussistenza al di fuori degli schemi tradizionali (scuola, lavoro, famiglia), schemi che quei giovani avvertivano come troppo "conservatori". Penso a voci come Bob Dylan, Jefferson Airplane, Doors. Questa "rivoluzione" musicale, culturale, funziona fino a un certo punto, perché ovviamente nessun intellettuale o rock star fece una rivoluzione vera e propria. Ebbe un qualche impatto sui diritti civili, sulla rivoluzione sessuale, sulla cultura delle droghe. Certamente non riuscì a fermare la guerra. E certamente non fermò il capitalismo. 


\section{S.A.}

Per ricollegarci al discorso sulla letteratura italiana contemporanea, mi sembra interessante il contrasto con quanto avviene in molti romanzi di oggi che sembrano suggerire il contrario e propongono una visione talvolta nostalgica dei decenni precedenti alla caduta del muro di Berlino. Molti dei romanzi più celebrati, penso ad esempio a Works, di Vitaliano Trevisan, o Ipotesi di una sconfitta, di Giorgio Falco, sottintendono un contrasto fra il presente e il passato del mondo del lavoro italiano. Si può pensare inoltre a un romanzo come Acciaio, di Silvia Avallone, che rievoca il passato glorioso delle acciaierie di Piombino contrapponendolo a un presente in cui la figura del lavoratore ha perduto la possibilità si sentirsi parte di un progetto politico che vada al di là della sua individualità, col risultato che le sue ambizioni si riducono alla stabilità del posto lavoro e a uno stipendio che non lo faccia sentire escluso dal benessere materiale che vede intorno a sé. Dal punto di vista degli imprenditori, si può evocare il romanzo che ha vinto il premio strega nel 2011, Storia della mia gente, di Edoardo Nesi, che racconta il declino del distretto tessile pratese fatto di aziende a conduzione familiare che prosperavano nell'epoca precedente all'attuale fase di globalizzazione. Uno dei contributi ospitati in questo volume si intitola "C'era una volta la fabbrica" e presenta appunto una rassegna di romanzi contemporanei che mettono in contrapposizione lo stato presente del mondo del lavoro con quello degli anni Sessanta. È curioso vedere come quello stesso passato che la generazione dei ventenni di allora vedeva come inaccettabile e che in letteratura ha prodotto opere come Vogliamo tutto, di Nanni Balestrini, che raccontava la ribellione dell'operaio massa, per non parlare dei romanzi di Ottiero Ottieri e Paolo Volponi... ecco, è curioso che quell'epoca adesso venga rievocata con nostalgia. Si tratta di un fenomeno che ha un riscontro anche da un punto di vista politico, se pensiamo a quanti partiti o movimenti anche solo d'opinione negli ultimi anni (diciamo a partire dalla crisi del debito del 2011) abbiano coltivato una sorta di nostalgia della "prima repubblica".

F.M.

Avverto anch'io una differenza fra il periodo che stavo descrivendo poco fa, cioè l'epoca successiva al boom, e il periodo attuale. Nel primo caso alludevo a una generazione che per qualche ragione si ribellava contro i padri, e in generale contro l'establishment; oggi c'è invece un'ingiustizia inter-generazionale reale, ma per qualche ragione facciamo più fatica a far passare questo messaggio. E questo si ricollega a quello che dice lei: c'è esattamente questo richiamarsi a 
una società che funzionava meglio negli anni Settanta e negli anni Ottanta, senza avvertire che l'abbiamo fatta funzionare meglio sottraendo risorse a chi sarebbe venuto dopo. E forse non ce ne siamo voluti rendere conto. Io l'ho vissuto anche da un punto di vista personale: genitori e parenti increduli per la fatica che fanno oggi i giovani (e che ho fatto io) per trovare lavoro, mentre ai loro tempi non c'era neanche bisogno di laurearsi e spesso già c'era qualcuno che ti voleva assumere. Ecco, probabilmente la classe politica degli anni ' $70 \mathrm{e}$ '80 ha "comprato" una vita semplificata per quella generazione, ma le misure dirigiste e assistenzialiste con cui hanno fatto questo, a forza di accumularsi, sono diventate un fardello che oggi pesa sui figli. Un fardello di cui è difficile liberarsi, senza un grande coraggio politico che al momento è tragicamente assente.

S.A.

Questo ci porta a un altro dei problemi del nostro tempo che compare, quantomeno in filigrana, anche in alcuni romanzi del lavoro, ovvero il problema del disallineamento tra domanda e offerta di lavoro; peraltro uno dei temi centrali del saggio di Ventura, Teoria della classe disagiata.

F.M.

Il libro di Ventura secondo me funziona abbastanza bene come fotografia, ma difetta sul lato della spiegazione, che poi è quanto servirebbe per dare all'analisi un valore scientifico. Un elemento ce lo siamo detto. Ovvero, se una generazione ha vissuto sulle spalle della successiva è evidente che delle risorse sono state sottratte alla seconda, che è costretta a vivere di mancette dai nonni, oltre che dallo Stato. Si accumula un forte debito che finisce per ricadere sulle imprese, e quindi sulla creazione di posti di lavoro. Ventura discorre in lungo e in largo sul disallineamento tra le ambizioni dei giovani, espresse nei percorsi accademici che scelgono, e le reali possibilità di trovare un'occupazione che rifletta quelle ambizioni. Mi sorprende che non veda questa parte della spiegazione.

C'è un'altra parte della spiegazione, più complessa, che ha a che fare con la nostra istruzione scolastica e universitaria. D'istinto mi verrebbe da essere in disaccordo con Ventura e dire che non è vero che con un dottorato in filosofia o un titolo di studi in materia umanistiche non si trova lavoro. Mi si chiederà: e allora a quale lavoro si può aspirare? Questo è il punto, le materie umanistiche sono caratterizzate dal fatto che è difficile dire a priori in quali lavori possono essere spese. Se uno studia medicina posso prevedere che farà il medico, se stu- 
dia legge posso prevedere che farà l'avvocato, ma se studia filosofia è un po' più difficile prevedere quale sarà la sua professione. Questo non significa che non ci siano delle opzioni, e allora come fare a identificarle? A mio parere occorre trovare il modo di connettere maggiormente il mondo dell'impresa a quello dell'istruzione e dell'accademia. Se tu parli con un recruiter, un HR Manager, un Responsabile Risorse Umane, saprà senz'altro tracciarti un profilo delle competenze (o skill, come si dice più spesso) che trova normalmente in un laureato in letteratura o in filosofia, e soprattutto di come è in grado di impiegarle. Ma questo tipo di informazione non arriva allo studente di filosofia o letteratura, e certamente nemmeno al liceale. Io ho studiato filosofia, sono un Ventura che non ha scritto un libro sull'attualità della mia generazione. Nel mio percorso mi avrebbe aiutato molto confrontarmi con un professionista della comunicazione o delle risorse umane, magari avendo la possibilità di fare un corso facoltativo di management. Sarebbe stato utile per chiarire quelle che erano le mie possibilità. Un esperto mi avrebbe spiegato che una laurea umanistica tende a potenziare le cosiddette "soft skills", tra cui la capacità di ragionare su un problema, di leggere e comprendere testi complessi, di comunicare correttamente, capacità che, se coltivate opportunamente, possono essere utili in un ambiente di lavoro. Tutto questo l'ho scoperto più tardi da solo, e non penso che il mio sia un caso unico. Naturalmente, lo studente di filosofia che è del tutto all'oscuro delle sue possibilità professionali tenderà a intestardirsi sulla carriera accademica, e svilupperà un senso di sconforto nel momento in cui non riuscirà a raggiungere quest'obiettivo. Non è vero che con le lauree umanistiche non si fa nulla, o in ogni caso, se le persone non ci fanno nulla, è perché non esiste un contesto che è in grado di renderle produttive. Non sono risposte che trovi in un libro.

\section{S.A.}

Vuol dire, quindi, che il problema è principalmente istituzionale, il che ci riporta al tema del disallineamento tra formazione e mercato del lavoro. Questo fenomeno viene spesso attribuito alla forma di capitalismo in cui è calato il nostro presente e che molti chiamano "neoliberismo", intendendo con questo una forma estremizzata di capitalismo che sottrae risorse al settore pubblico prediligendo una competizione senza regole fra attori privati. Una sorta di mondo Hobbesiano che si trova evocato nello stesso saggio di Ventura e di cui troviamo ampio riflesso nei romanzi a tema economico. 
F.M.

Chi la pensa in questo modo, nel parlare di "capitalismo" si riferisce - che ne sia cosciente o no - a un intreccio complesso tra stato e mercato, tra istituzioni e imprese. Se capitalismo significa regolamentazioni distorsive, imprese impegnate nella ricerca di rendite molto più di quanto siano interessate a innovare $\mathrm{e}$ cercare soluzioni a beneficio delle persone, beh, è difficile non essere d'accordo. Ma il capitalismo non penso sia questo, e quella non è una spiegazione, perché non compie lo sforzo di isolare i fattori che spingono in una direzione piuttosto che in un'altra (cioè stato e mercato).

\section{S.A.}

Questo vuol dire che "capitalismo" sarebbe un termine ombrello che dice poco di come davvero funziona l'economia moderna?

F.M.

È sicuramente un termine che porta a dei fraintendimenti (ecco perché sarebbe preferibile parlare di "società commerciale", o semplicemente di mercato). E il fatto che "capitalismo" sia un termine che si presta a equivoci non ci aiuta a spiegare la ragione dell'ostilità degli intellettuali. Secondo me una spiegazione del genere è assente anche in Kahan, che documenta la persistenza di quest'ostilità nei confronti del capitalismo, ma una spiegazione della persistenza del pregiudizio non credo sia qualcosa che possiamo chiedere al suo libro. Un'altra parte della spiegazione che stiamo cercando, e che mi sento di suggerire, è la difficoltà ad afferrare l'idea del mercato come ordine spontaneo. È un'idea cruciale, perché se non si comprende questo punto ci si convince che la società capitalistica sia basata sul dominio, sul controllo, e quindi sulla privazione di libertà.

\section{S.A.}

Cosa si intende per "ordine spontaneo"?

F.M.

Tutti conosciamo la metafora di Adam Smith, la "mano invisibile". È una metafora che purtroppo è stata spesso male interpretata, come se volesse significare la teorizzazione di una qualche forza misteriosa che orienterebbe la società verso un certo risultato. In realtà, sposare l'idea della società come "ordine spontaneo" significa essenzialmente due cose, una di carattere descrittivo, l'altra di carattere normativo. La prima è l'idea che le istituzioni 
sociali - intese come norme formali o informali che gli individui seguono nell'interagire in società - sono il frutto di un processo di scoperta dal basso, essenzialmente non guidato, in cui alcuni costumi sono premiati perché permettono meglio di altri di ridurre l'incertezza. Gli esempi classici sono il linguaggio, il denaro, i prezzi dei beni. La seconda idea è che è preferibile che questo processo di scoperta sia lasciato il più possibile libero da interferenze. Fare diversamente significa cadere nell'illusione che le conoscenze rilevanti possano essere accentrate nelle mani di pochi decisori, laddove esse sono per definizione disperse (come teorizzò Friedrich Hayek).

Questa è un'altra parte della spiegazione che stavamo cercando. Nel momento in cui chiedono meno mercato, gli intellettuali stanno implicitamente assumendo che le scelte politiche possano orientare la società civile verso risultati migliori di quanto farebbe quel processo di scoperta dal basso. Ma ordine spontaneo significa anche che la regola stabilita dal legislatore e imposta sulla società civile altera in modi imprevedibili il comportamento degli individui, perdendosi in una rete complessa di scelte e di azioni individuali, e può in tal modo portare a risultati diversi da quelli sperati. Se il legislatore riduce il prezzo di un bene di prima necessità durante una calamità, disincentiverà la produzione di quel bene, al quale di conseguenza diventerà più difficile accedere. Ho la sensazione che gli studenti, e quindi gli intellettuali di domani, siano invece cresciuti nella convinzione che le idee, i principi, la "cultura" e i valori siano in grado di plasmare la società civile, possano cioè essere applicati producendo dei risultati prevedibili.

\section{S.A.}

Traducendo Mind vs Money le sarà capitato di notare forse con maggiore immediatezza come in molta fiction contemporanea, quale che sia lo specifico codice espressivo, emerga l'antagonismo fra intellettuali e capitalismo descritto da Kahan. Quale esperienza di spettatore o lettore potrebbe condividere in questo senso?

F.M.

Rispondere a questa domanda è più difficile di quello che sembra. Come è noto, gli intellettuali tendono a leggere film e serie TV come pro o anticapitalisti a seconda del proprio orientamento (come fece Žižek con The Dark Knight Rises) ${ }^{3}$. C'è il rischio di forzare quelle che sono soltanto delle storie. Ad esempio,

3. Stavoj Žižek, "The Politics of Batman”, New Statesman, 23 agosto 2012, https:/ / www.newstatesman.com/culture/culture/2012/08/slavoj- $\%$ C5\%BEi $\%$ C5\%BEekpolitics-batman 
Una poltrona per due è un film anticapitalista o è soltanto una storia? La mia sensazione è che film o serie TV con un taglio così netto non siano molte. Ma non serve che ci sia una presa di posizione esplicita contro il capitalismo. Possono esserci rappresentazioni negative del denaro, della finanza (Drag Me to Hell di Sam Raimi, The Wolf of Wall Street di Scorsese, o la chiacchieratissima serie La casa di carta), della figura dell'imprenditore (penso a The Founder, il recente film su Ray Kroc, l'ideatore di McDonald's). E questi giudizi sono qualcosa di sedimentato, quasi dei luoghi comuni, non sempre il risultato di un'elaborazione esplicita o di un intento di denuncia. Quindi sono ancora più profondi.

\section{S.A.}

Tutto ciò sembra contrastare con l'insegnamento che ci hanno lasciato i teorici della Scuola di Francoforte, il cui nucleo fondamentale gode ancora oggi di una certa popolarità fra chi studia la formazione e diffusione delle ideologie.

F.M.

La Scuola di Francoforte è uno degli indirizzi di pensiero più elitisti ai quali io possa pensare. Si nutre di una distinzione tra chi capisce come va il mondo, l'intellettuale, e la massa che invece non comprende. Quando penso a Horkheimer e Adorno mi viene sempre in mente un passaggio della Dialettica dellilluminismo all'interno di una disquisizione sull'industria culturale. È una sorta di vignetta in cui i due raccontano l'esperienza del lavoratore che, terminata la sua giornata di lavoro, va al cinema a vedere i cartoni animati di Paperino. Ecco, nel ridere delle disavventure di Paperino - di Paperino che prende le botte in testa - il lavoratore in realtà sta inconsapevolmente ridendo della propria condizione. Facendogli accettare con rassegnazione la propria vita di ingiustizie, il capitalismo, grazie all'industria culturale, ha completato la propria missione, ed è finalmente riuscito a soggiogare la classe operaia. Come dicevo, non riesco a pensare a nulla di più elitista. Scelte banali come andare al cinema per vedere un cartone animato - e quindi comprare un dentifricio, andare in vacanza, acquistare una macchina, ecc. - sono in realtà qualcosa di subito, cioè non è mai una scelta realmente libera.

Non so se quest'esempio ci porta più vicini alla spiegazione, però certamente ci aiuta a leggere meglio la frattura fra i due mondi, intellettuali e capitalismo, e l'incomprensione da parte dei primi di quello che abbiamo chiamato "ordine spontaneo". Se la società è un ordine spontaneo, non c'è nessun intento malevolo nella proiezione di Paperino, ma semplicemente lo sforzo di un'impresa di offrire 
qualcosa che al pubblico piacerà. Non a caso la maggior parte dei prodotti culturali sono anticapitalisti, perché evidentemente alle persone piacciono di più.

\section{S.A.}

Parlavamo della rappresentazione mediatica o letteraria della figura dell'imprenditore. È un fatto che in Italia essa sia, il più delle volte, caratterizzata da un'aperta ostilità, un sentimento che si rivela già nel termine con cui tradizionalmente lo si è denotato: "padrone". È anche un fatto che l'imprenditoria italiana è spesso associata, nella sensibilità comune, a politiche dal carattere reazionario, pur con qualche notevole eccezione. Non sarà forse che la storia del connubio fra potere politico, alta finanza e grandi imprese, spesso oligarchiche, ha finito per alimentare un'immagine parassitaria e antisociale dell'imprenditore? Inoltre, ci si potrebbe chiedere se tutto ciò non sia anche il risultato del fatto che in Italia di imprese innovative se ne son viste poche nel corso degli ultimi decenni, mentre di rendite di posizione e industrie protette è pieno il paese.

F.M.

Quello della rappresentazione critica dell'imprenditore è un fenomeno non solo italiano, ma che in Italia assume connotati particolari per un semplice motivo: la figura dell'imprenditore virtuoso non è qualcosa che abbiamo imparato a conoscere. Conosciamo meglio l'imprenditore nei termini in cui lo descrive lei, quello che si fa strada in virtù dei suoi legami con la politica. Un'impresa virtuosa, come una grande multinazionale, tendiamo a conoscerla per i prodotti che troviamo nei negozi, più che per i meriti imprenditoriali, che certamente non finiscono sui giornali (se non qualche rubrica o testata specialistica). E quindi, quando pensiamo all'imprenditore, a cosa pensiamo? A un settore altamente politicizzato e regolamentato come la finanza (lo stesso Ventura si rifà al romanzo di Zola sul Denaro), pensiamo all'Ilva, ad Alitalia, allo speculatore edilizio di Le mani sulla città, non esattamente un benefattore bensì una figura che vive alle spalle degli altri (come sottolineato dal palazzo che crolla in una delle prime sequenze del film). E c'è della verità in queste rappresentazioni, perché il nostro è un contesto che crea incentivi a cercare il favore del potere, anziché ad innovare o investire in ricerca e sviluppo. Torniamo al punto di prima, dobbiamo far passare un messaggio diverso, ossia che nel momento in cui chiediamo un contesto che incentivi l'impresa virtuosa e disincentivi quella parassitaria, stiamo per l'appunto chiedendo più mercato, più capitalismo. 
Torniamo quindi allidea del mercato che si "autoregola", un punto su cui vorrei fare un'osservazione. Smettiamo di vedere il mercato come qualcosa che ci sovrasta e impariamo a capire che del mercato fanno parte tutti gli ignoti che la mattina si svegliano, vanno al lavoro, scelgono cosa comprare, a quali prodotti culturali dar retta, quali idee far circolare. Il mercato, per come lo vedevano gli economisti austriaci (e io mi ci ritrovo), non è altro che la società civile stessa. È un sistema fatto non soltanto di scambi commerciali, benché ne siano ovviamente una parte essenziale, ma anche di associazioni, di enti no-profit, di circolazione di idee, e questi input hanno un'eco nel mercato in senso stretto, si riflettono nelle decisioni e nelle strategie delle imprese, nelle scelte di produzione, di marketing, di comunicazione. Pensiamo a una qualsiasi impresa - anche tra quelle più "cattive", ad alto impatto ambientale, o l'industria del tabacco: avrà sicuramente in cantiere un qualche piano di sostenibilità, un progetto di dialogo con il territorio (può essere il risanamento di un terreno, il recupero di un edificio in disuso, una campagna di sensibilizzazione su una tematica d'interesse sociale). Ciò accade esattamente in virtù del fenomeno che descrivo. È il mercato in senso stretto (le scelte di produzione e le strategie d'impresa) che incorpora nelle proprie decisioni i segnali dal mercato in senso più ampio (le idee, le opinioni, i valori). Un'impresa non può non tenere conto di questi segnali senza compromettere la propria brand reputation.

\section{S.A.}

Eppure, una delle raccomandazioni per cui la cosiddetta scuola "neoliberale" è più nota è che le imprese dovrebbero avere come unico fine quello di massimizzare il profitto per i propri azionisti. Ciò si pone in diretto contrasto con una cultura d'impresa che in Italia è sempre stata più apprezzata, ovvero quella dell'impresa che radicandosi in un territorio contribuisce attivamente al suo sviluppo erogando forme di welfare o promuovendo attività culturali. Come mai in Italia prevale questo modello, mentre in molti altri paesi si lascia che le imprese si concentrino sulla loro produzione e si assegna invece alla politica il compito di trasformare la ricchezza prodotta dalle imprese in sviluppo territoriale e benessere per la collettività?

\section{F.M.}

A mio avviso la differenza tra questi due modelli, in Italia come altrove, si sta assottigliando sempre di più. È vero che il profitto è, e rimarrà sempre, la cartina di tornasole del successo di un'impresa, ma è anche vero che la via per il profitto 
è oggi un po' più complessa, e non può non tenere conto di ciò a cui accennavo poco fa. Si tratta, in effetti, di un effetto indiretto del benessere creato dal capitalismo. Nello scegliere quale prodotto acquistare, possiamo permetterci di prendere in considerazione non soltanto il risparmio o la qualità "nuda" dell'oggetto, ma anche cosa c'è dietro la sua produzione, se l'impresa che lo ha prodotto segue un'etica del lavoro, se attua policy inclusive o environment-friendly. E il mercato, come dicevo, registra questo segnale. È un fenomeno che non ha solo risvolti positivi, naturalmente, perché tra i segnali che le imprese hanno registrato negli ultimi anni c'è anche la psicosi da olio di palma, che è sparito da quasi tutti i prodotti. Domani potrebbe esserci la psicosi del 5G. Il mercato è un sistema imperfetto, però dobbiamo scegliere: o accettiamo le imperfezioni di un sistema che è molto bene in grado di integrare preferenze e valori del pubblico nelle proprie strategie, oppure ci autocondanniamo agli esiti ben più gravi - che non sono semplici imperfezioni - che derivano dalle interferenze dello Stato nel mercato.

$\mathrm{E}$ in questa concezione allargata del mercato possiamo in parte recuperare un'idea espressa da Kahan nella parte conclusiva del libro. Secondo Kahan, il ruolo che gli intellettuali possono e devono avere nei confronti del capitalismo è un ruolo "correzionale". Devono esibire e argomentare a favore di quelle idee e quei valori che il mercato tenderebbe a tralasciare, essendo votato soltanto al profitto. A mio avviso le imprese non pensano "solo" al profitto, se pensare solo al profitto significa ignorare la propria responsabilità nei confronti della società. Come dicevo, io non vedo questa polarità tra commercio e valori. Però fa bene Kahan a sottolineare che il sistema capitalistico non ha nessuna allergia per il lavoro degli intellettuali e che ha anzi bisogno di una riflessione su tematiche di importanza sociale (l'ambiente, l'eguaglianza di genere, l'inclusione sociale). Ma tutto questo lo si comprende meglio se usciamo dalla dicotomia capitalismo vs. intellettuali. Semplicemente, la società si regge su un principio di divisione del lavoro, e non è banale far parlare mondi altamente specializzati e differenti come quello imprenditoriale-manageriale e quello culturale. Se riuscissimo a capire come farlo, avremmo forse prodotto un grande beneficio sociale, oltre a una classe intellettuale meno alienata.

Intervista a cura di Stefano ADAMO

Università di Banja Luka 